\title{
2. SYNOPSIS
}

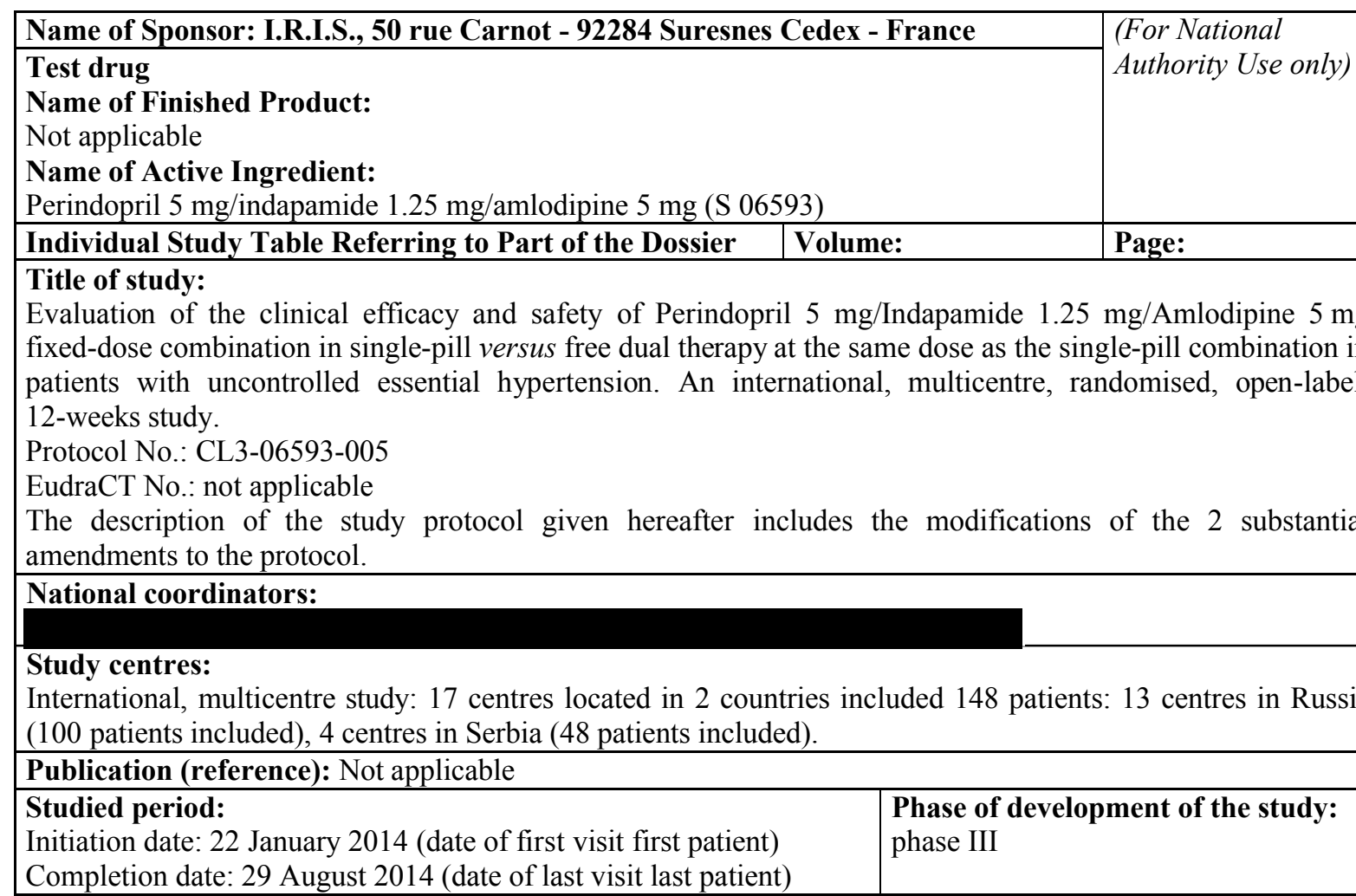

\section{Objectives:}

The objective of this study was to evaluate the clinical efficacy and safety of fixed-dose combination perindopril $5 \mathrm{mg}$ /indapamide $1.25 \mathrm{mg}$ /amlodipine $5 \mathrm{mg}$ in single-pill versus free dual monocomponents, perindopril $5 \mathrm{mg}$ /indapamide $1.25 \mathrm{mg}$ and amlodipine $5 \mathrm{mg}$ over 12 weeks, in patients having an uncontrolled hypertension defined by Systolic Blood Pressure (SBP) $\geq 140$ and $<160 \mathrm{mmHg}$ and Diastolic Blood Pressure (DBP) $\geq 90$ and $<100 \mathrm{mmHg}$ under on-going treatment (anti-hypertensive monotherapy at maximal dose or either by a single dose of dual therapy other than study treatment).

\section{Methodology:}

International, multicentre, randomised, open-label, controlled phase III study, over a 12-week treatment period, comparing single-pill combination of fixed dose combination of perindopril $5 \mathrm{mg} / \mathrm{indapamide}$ $1.25 \mathrm{mg}$ /amlodipine $5 \mathrm{mg}$ versus free dual therapy by fixed dose combination of perindopril $5 \mathrm{mg} / \mathrm{indapamide}$ $1.25 \mathrm{mg}$ and by amlodipine $5 \mathrm{mg}$ given separately, in patients with an uncontrolled hypertension (defined by $\mathrm{SBP} \geq 140 \mathrm{mmHg}$ and $<160 \mathrm{mmHg}$ and DBP $\geq 90 \mathrm{mmHg}$ and $<100 \mathrm{mmHg}$ at 2 different visits (selection and inclusion)).

This study was performed in strict accordance with Good Clinical Practice including the archiving of essential documents.

\section{Number of patients:}

Planned: 150 patients, 75 patients in each treatment group.

Included: 148 patients, 75 patients in the perindopril $5 \mathrm{mg} /$ indapamide $1.25 \mathrm{mg} /$ amlodipine $5 \mathrm{mg}(\mathrm{Per} / \mathrm{Ind} / \mathrm{Aml})$ group, 73 patients in the perindopril $5 \mathrm{mg} /$ indapamide $1.25 \mathrm{mg}+$ amlodipine $5 \mathrm{mg}$ (Per/Ind + Aml) group.

\section{Diagnosis and main criteria for inclusion:}

Men or women of any ethnic origin $\geq 18$ years old or legal national majority, with confirmed essential uncontrolled hypertension, already treated by anti-hypertensive monotherapy at maximal dose or by a single dose of dual therapy other than study treatment (perindopril or indapamide or amlodipine), with no contraindications to treatment with perindopril, indapamide, or amlodipine.

\section{Test drug:}

S06593 fixed combination of perindopril $5 \mathrm{mg}$ /indapamide $1.25 \mathrm{mg} /$ amlodipine $5 \mathrm{mg}$, administered orally as one tablet daily before breakfast.

Batch Nos.: L0042920, L0050304. 
Comparator (Reference product and/or placebo):

Perindopril $5 \mathrm{mg}$ /indapamide $1.25 \mathrm{mg}+$ amlodipine $5 \mathrm{mg}$, administered orally as one tablet daily each one, in the morning before breakfast.

\section{Duration of treatment:}

Run-in period: one week, where all patients had to receive their current treatment for hypertension.

Treatment period: 12 weeks, including W4, W8, and W12 (final) visits.

\section{Criteria for evaluation:}

Efficacy measurements:

- Supine and standing blood pressure measured using an OMRON device at all visits and at withdrawal visit, on the same arm.

- Response criteria: rate of patients with blood pressure normalised (SBP $<140 \mathrm{mmHg}$ and DBP $<90 \mathrm{mmHg}$ ), and/or decrease of SBP $\geq 20 \mathrm{mmHg}$, and/or decrease of DBP $\geq 10 \mathrm{mmHg}$ from baseline, measured at W12.

\section{Safety measurements:}

- Adverse events at each visit (selection, inclusion, W4, W8, and W12).

- Laboratory test:

- $\beta$-hCG pregnancy test (blood or urine test) at inclusion.

- Complete laboratory examinations, at inclusion and W12: blood biochemistry (sodium, potassium, chloride, calcium, uric acid, urea, creatinine, fasting blood glucose levels, total protein, triglycerides, total cholesterol, ASpartate (Amino) Transferase (ASAT), ALanine (Amino) Transferase (ALAT), Gamma-Glutamyl Transferase (GGT)), haematology (haemoglobin, haematocrit, erythrocytes, neutrophils, basophils, eosinophils, lymphocytes, monocytes, leucocytes and platelets), and urine check for proteinuria (at inclusion, if applicable).

- Simplified laboratory tests at W4: creatinine, creatinine clearance, uric acid and potassium.

- Physical examination: weight at selection, W4 and W12, and height at selection.

- Vital signs: orthostatic hypotension, and heart rate at each visit.

- 12-lead electrocardiogram at inclusion and W12.

\section{Statistical methods:}

Study outcome and safety analysis: descriptive statistics were provided.

Efficacy analysis:

The analysis Set used for the main analysis of the primary endpoints was the Full Analysis Set (FAS): based on the intention-to-treat principle, this Set correspond to randomised patients having received at least one dose of study treatment and having a value at baseline and at least one post-baseline value for office supine SBP and DBP over W0-W12 period.

For all primary and secondary endpoints described hereafter, the treatment effect was estimated, as well as its accuracy: estimate of the difference, standard error of the estimate and two-sided $95 \%$ confidence interval of the estimate.

\section{Primary endpoints:}

- Office supine SBP and DBP ( $\mathrm{mmHg}$ ), mainly expressed in term of the change from baseline to last post baseline value over the W0-W12 period.

Secondary expressions are:

- Value at baseline and at each post-baseline visit (W4, W8, W12).

- Last post baseline visit value over the W0-W12 period.

- Response to treatment expressed mainly as the last post-baseline value (over the W0-W12 period).

Secondary endpoints: office supine SBP and DBP (mmHg), and the office standing SBP and DBP at $3 \mathrm{~min}$ $(\mathrm{mmHg})$, mainly expressed as the change from baseline to last post baseline value at each post-baseline visit, response to anti-hypertensive treatment expressed as the rate of responders at last post-baseline value at each post-baseline visit, and office supine blood pressure control mainly expressed as the rate of patients at last post-

baseline value at each post-baseline visit. 


\begin{tabular}{|c|c|c|c|c|}
\hline \multicolumn{5}{|c|}{$\begin{array}{l}\text { SUMMARY - CONCLUSIONS } \\
\text { DISPOSITION OF PATIENTS AND ANALYSIS SETS }\end{array}$} \\
\hline Status & & Per/Ind/Aml & Per/Ind + Aml & All \\
\hline Included/randomised & n (\%) & $75(100)$ & $73(100)$ & $148(100)$ \\
\hline In compliance with the protocol & $\mathrm{n}(\%)$ & $59(78.7)$ & $57(78.1)$ & $116(78.4)$ \\
\hline With a protocol deviation before or at inclusion & $\mathrm{n}(\%)$ & $16(21.3)$ & $16(21.9)$ & $32(21.6)$ \\
\hline Withdrawn due to & n (\%) & $5(6.7)$ & $9(12.3)$ & $14(9.5)$ \\
\hline Protocol deviation & $\mathrm{n}(\%)$ & $3(4.0)$ & $7(9.6)$ & $10(6.8)$ \\
\hline Adverse event & $\mathrm{n}(\%)$ & $1(1.3)$ & $1(1.4)$ & $2(1.4)$ \\
\hline Non-medical reason & $\mathrm{n}(\%)$ & $1(1.3)$ & - & $1(0.7)$ \\
\hline Other protocol withdrawal criteria & $\mathrm{n}(\%)$ & - & $1(1.4)$ & $1(0.7)$ \\
\hline Lack of efficacy & $\mathrm{n}(\%)$ & - & - & - \\
\hline Lost to follow-up & $\mathrm{n}(\%)$ & - & - & - \\
\hline Completed & n (\%) & $70(93.3)$ & $64(87.7)$ & $134(90.5)$ \\
\hline In compliance with the protocol & $\mathrm{n}(\%)$ & $58(77.3)$ & $54(74.0)$ & $112(75.7)$ \\
\hline With a protocol deviation after inclusion & $\mathrm{n}(\%)$ & $12(16.0)$ & $10(13.7)$ & $22(14.9)$ \\
\hline
\end{tabular}

n: number of patients affected; \%: expressed as percentage of the patients from the Included/Randomised Set

A total of 148 patients were included and randomly assigned to one of the two treatment groups, with a well-balanced distribution achieved: 75 patients in the Per/Ind/Aml group, and 73 patients in the Per/Ind + Aml group. Of them, 14 patients withdrew the study (5 patients in the Per/Ind/Aml group versus 9 patients in the Per/Ind + Aml group). The main reason for patient's withdrawal was protocol deviations: 10 patients, 3 patients versus 7 patients, respectively. The number of patients withdrawn for adverse event was low in both treatment groups (1 patient in each treatment group). Finally, 134 patients completed the study (70 patients and 64 patients, respectively). The number of patients affected by protocol deviations at inclusion was similar in both treatment groups (16 patients in both treatment groups). Patients reported mainly protocol deviations in relation with the blood pressure values (12 patients versus 13 patients, respectively). Regarding protocol deviations during the study, no relevant between-group difference was observed (17 patients versus 16 patients, respectively). They were mainly in relation with the sampling form (8 patients versus 6 patients, respectively).

\section{BASELINE CHARACTERISTICS}

In the Randomised Set, patients were in average $56.4 \pm 10.8$ years old, and an important part of the randomised patients $(43.9 \%)$ were at least 60 years old, with a higher frequency reported in the Per/Ind/Aml group than in the Per/Ind + Aml group: 35 patients, $46.7 \%$ versus 30 patients, $41.1 \%$, respectively. About half of the patients were women (78 patients, $52.7 \%$ ). The mean BMI was $27.8 \pm 3.1 \mathrm{~kg} / \mathrm{m}^{2}$ (ranging from 16.0 to $32.2 \mathrm{~kg} / \mathrm{m}^{2}$ ). All patients except one (considered as having a protocol deviation) had a BMI $<32 \mathrm{~kg} / \mathrm{m}^{2}$.

All patients presented at selection an essential hypertension, in average since $98.9 \pm 84.3$ months. A family history of hypertension was reported in most of the patients: 104 patients, $70.3 \%$. A complication of the hypertension was reported in 4 patients ( 2 peripheral artery disease in the Per/Ind/Aml group and 2 heart disease in the Per/Ind + Aml group). No relevant between-group difference was observed.

Other medical history in relation with hypertension was dyslipidaemia with a higher frequency reported in the Per/Ind/Aml group than in the Per/Ind + Aml group (40 patients, 53.3\% versus 32 patients, $43.8 \%$, respectively), and cardiovascular disease (4 patients, $5.3 \%$ in the Per/Ind/Aml group versus 5 patients, $6.9 \%$, respectively) or diabetes mellitus (4 patients, $5.3 \%$ versus 4 patients, $5.5 \%$, respectively) reported with similar frequency in both treatment groups.

All patients received at least one treatment for essential hypertension before inclusion in the study, mainly agents acting on the renin-angiotensin system (93.3\% versus $98.6 \%$, respectively), and diuretics $(61.3 \%$ versus $68.5 \%$, respectively).

Regarding risk factors, smoking habit was reported in $18.2 \%$, and alcohol habit consumption in $23.7 \%$, with a higher rate reported in the Per/Ind/Aml than in the Per/Ind + Aml group (26.7\% versus 20.6\%, respectively).

No relevant between-group difference was detected for vital signs. The supine heart rate was in average $76.6 \pm 10.9$ beats per minute (bpm) (ranging from 55.0 to $112.5 \mathrm{bpm})$. 


\section{SUMMARY - CONCLUSIONS (Cont'd)}

\section{BASELINE CHARACTERISTICS (Cont'd)}

All patients had an ECG performed at baseline. Among them, 53 patients, $35.8 \%$ had an ECG abnormality, of which one in the Per/Ind + Aml group was considered as clinically significant.

Blood pressure parameters at inclusion were similar at baseline, in both treatment groups, in the Randomised Set. The supine SBP and DBP were in average: $149.1 \pm 4.7 \mathrm{mmHg}$ and $94.1 \pm 3.1 \mathrm{mmHg}$, with no relevant between group-difference (SBP: $149.1 \pm 4.9 \mathrm{mmHg}$ in the Per/Ind/Aml group and $149.1 \pm 4.6 \mathrm{mmHg}$ in the Per/Ind + Aml group, DBP: $94.1 \pm 3.1 \mathrm{mmHg}$ and $94.2 \pm 3.3 \mathrm{mmHg}$, respectively).

Demographic and other baseline characteristics in the FAS and the PPS were in the same line.

\section{EXTENT OF EXPOSURE}

The mean treatment duration was $82.2 \pm 13.8$ days over the W0-W12 period, in the FAS. The mean treatment compliance was good: $97.6 \pm 6.4 \%$, and all patients but one had an overall compliance between $80 \%$ and $120 \%$. Neither between-group difference was detected, nor between-group difference when each pill was considered.

\section{EFFICACY RESULTS \\ Primary endpoints}

\section{- Office supine SBP/DBP over the W0-W12 period}

The decrease in the office supine SBP was observed in both treatment groups from baseline to the last postbaseline value (End), whereas these patients were already under on-going anti-hypertensive therapy before entry in the study (monotherapy at maximal dose or a single dose of dual therapy other than study treatment). The changes were similar in both treatment groups, in the FAS (see table hereafter). Same trends were observed in the PPS.

Office supine SBP - Change from baseline to last post-baseline value Comparison between groups during the study period (W0-W12) - FAS $(\mathrm{N}=144)$

\begin{tabular}{|c|c|c|c|}
\hline Supine blood pressure & & $\begin{array}{c}\text { Per/Ind/Aml } \\
(\mathrm{N}=74)\end{array}$ & $\begin{array}{c}\text { Per/Ind + Aml } \\
(\mathbf{N}=70)\end{array}$ \\
\hline \multicolumn{4}{|l|}{ SBP } \\
\hline \multirow[t]{5}{*}{ Baseline } & $\mathrm{n}$ & 74 & 70 \\
\hline & Mean \pm SD & $149.10 \pm 4.89$ & $149.01 \pm 4.66$ \\
\hline & $95 \% \mathrm{CI}$ & {$[147.97 ; 150.23]$} & {$[147.90 ; 150.12]$} \\
\hline & Median & 148.75 & 149.75 \\
\hline & Min ; Max & $140.0 ; 160.0$ & $140.5 ; 157.5$ \\
\hline \multirow[t]{5}{*}{ End W12 - Baseline } & & 74 & 70 \\
\hline & Mean \pm SD & $-21.54 \pm 11.70$ & $-20.02 \pm 12.85$ \\
\hline & $95 \% \mathrm{CI}$ & {$[-24.25 ;-18.83]$} & {$[-23.09 ;-16.96]$} \\
\hline & Median & -21.00 & -20.75 \\
\hline & Min ; Max & $-50.0 ; 2.5$ & $-49.5 ; 23.0$ \\
\hline \multicolumn{4}{|l|}{ Statistical analysis } \\
\hline & \multirow{2}{*}{$\begin{array}{l}\text { E (SE) (1) } \\
95 \% \text { CI (2) }\end{array}$} & \multirow{2}{*}{\multicolumn{2}{|c|}{$\begin{array}{c}-1.42(1.84) \\
{[-5.07 ; 2.22]}\end{array}$}} \\
\hline & & & \\
\hline \multicolumn{4}{|l|}{ DBP } \\
\hline \multirow[t]{5}{*}{ Baseline } & $\mathrm{n}$ & 74 & 70 \\
\hline & Mean \pm SD & $94.09 \pm 3.00$ & $94.14 \pm 3.09$ \\
\hline & $95 \% \mathrm{CI}$ & {$[93.39 ; 94.78]$} & {$[93.41 ; 94.88]$} \\
\hline & Median & 93.75 & 94.50 \\
\hline & Min ; Max & $86.0 ; 104.5$ & $81.0 ; 101.0$ \\
\hline \multirow[t]{5}{*}{ End W12 - Baseline } & $\mathrm{n}$ & 74 & 70 \\
\hline & Mean \pm SD & $-15.31 \pm 7.80$ & $-14.81 \pm 8.98$ \\
\hline & $95 \% \mathrm{CI}$ & {$[-17.12 ;-13.50]$} & {$[-16.96 ;-12.67]$} \\
\hline & Median & -14.50 & -15.25 \\
\hline & Min ; Max & $-37.5 ;-1.5$ & $-40.5 ; 5.0$ \\
\hline \multicolumn{4}{|l|}{ Statistical analysis } \\
\hline & \multirow{2}{*}{$\begin{array}{l}\text { E (SE) (1) } \\
95 \% \text { CI (2) }\end{array}$} & \multicolumn{2}{|c|}{$-0.53(1.31)$} \\
\hline & & {$[-3.1$} & $06]$ \\
\hline \multicolumn{4}{|c|}{$\begin{array}{l}\text { : number of patients in each treatment group; } n: \text { number of observed values; End: last value posterior to } \\
\text { the first treatment intake; \%: }(n * 100 / N) \text {; (1) Estimate (Standard Error) of the difference in adjusted mean } \\
\text { changes from baseline to last post-baseline value until W12 single-pill combination - free dual therapy } \\
\text { using a General Linear Model with treatment, baseline and centre as covariates; (2) } 95 \% \text { Confidence } \\
\text { Interval of the estimate }\end{array}$} \\
\hline
\end{tabular}




\section{SUMMARY - CONCLUSIONS (Cont'd) \\ EFFICACY RESULTS (Cont'd)}

\section{- Response to treatment over the W0-W12 period}

The response to treatment for antihypertensive therapy was defined as the rate of patients with a blood pressure normalised (i.e. SBP $<140 \mathrm{mmHg}$ and $\mathrm{DBP}<90 \mathrm{mmHg}$ ), and/or a decrease of SBP $\geq 20 \mathrm{mmHg}$ from baseline, and/or decrease of DBP $\geq 10 \mathrm{mmHg}$ from baseline.

The response to the anti-hypertensive treatment at the last post-baseline value was observed for most of the patients in both treatment groups, and the frequency of responders was similar in both treatment groups: $89.2 \%$ in the Per/Ind/Aml group versus $87.1 \%$ in the Per/Ind + Aml group (Estimate of the between-group difference and its associated Standard Error E $(\mathrm{SE})=2.05(5.39) \%, 95 \%$ Confidence Interval $(\mathrm{CI})=[-8.51 ; 12.61])$, in the FAS.

Same trends were observed in the PPS.

\section{Secondary endpoints}

- Office supine SBP/DBP: change from baseline to last post-baseline value (W4 and W8) over the W0-W12 period

A decrease in the office supine SBP/DBP was observed in both treatment groups from baseline to each last post-baseline value at W4 and W8, and results were similar in both treatment groups. The changes were the following, showing that the full effect on the office supine SBP/DBP was reached at W4 and maintained until W12, in the FAS:

- W4: SBP $=-19.84 \pm 9.53 \mathrm{mmHg}$ in the Per/Ind/Aml group versus $-18.96 \pm 12.05 \mathrm{mmHg}$ in the Per/Ind + Aml group $(\mathrm{E}(\mathrm{SE})=-0.71(1.60) \mathrm{mmHg}, 95 \% \mathrm{CI}=[-3.88 ; 2.46])$, and $\mathrm{DBP}=-14.57 \pm 7.57 \mathrm{mmHg}$ versus $-14.67 \pm 9.47 \mathrm{mmHg}$, respectively $(\mathrm{E}(\mathrm{SE})=0.04(1.32) \mathrm{mmHg}, 95 \% \mathrm{CI}=[-2.57 ; 2.65])$.

- W8: SBP $=-20.71 \pm 10.43 \mathrm{mmHg}$ in the Per/Ind/Aml group versus $-23.59 \pm 12.10 \mathrm{mmHg}$ in the Per/Ind + Aml group $(\mathrm{E}(\mathrm{SE})=2.94(1.83) \mathrm{mmHg}, 95 \% \mathrm{CI}=[-0.67 ; 6.55])$, and $\mathrm{DBP}=-15.96 \pm 7.95 \mathrm{mmHg}$ versus $-17.13 \pm 8.86 \mathrm{mmHg}$, respectively $(\mathrm{E}(\mathrm{SE})=1.17(1.29) \mathrm{mmHg}, 95 \% \mathrm{CI}=[-1.38 ; 3.72])$.

\section{- Response to treatment at last post-baseline value (W4 and W8) over the W0-W12 period}

The response to the anti-hypertensive treatment at each last post-baseline value (at W4 and W8) was observed for most of the patients in both treatment groups, and was similar in both treatment groups. The high frequency of responders was observed at W4 and maintained until W12, in both treatment groups:

- W4: $89.2 \%$ in the Per/Ind/Aml group versus $82.9 \%$ in the Per/Ind + Aml group (E (SE) = $6.33(5.77) \%$, $95 \% \mathrm{CI}=[-4.98 ; 17.65])$.

- W8: $91.9 \%$ versus $92.9 \%$, respectively $(\mathrm{E}(\mathrm{SE})=-0.97(4.42) \%, 95 \% \mathrm{CI}=[-9.63 ; 7.70])$.

- Office supine blood pressure control at each last post-baseline value over the W0-W12 period

The control of the supine BP (defined as SBP $<140 \mathrm{mmHg}$ and DBP $<90 \mathrm{mmHg}$ ) was obtained in the majority of the patients in both treatment groups, at each last post-baseline value (at W4, W8, and W12).

The high rate of patients with a control of the supine BP was already obtained at W4 and maintained until $\mathrm{W} 12$, in both treatment groups, in the FAS:

- W4: 87.8\% in the Per/Ind/Aml group versus 78.6\% in the Per/Ind + Aml group (E (SE) = 9.27 (6.20) \%, $95 \% \mathrm{CI}=[-2.89 ; 21.43])$.

- $\mathrm{W} 8: 85.1 \%$ versus $88.6 \%$, respectively $(\mathrm{E}(\mathrm{SE})=-3.44(5.62) \%, 95 \% \mathrm{CI}=[-14.45 ; 7.57])$.

- $\mathrm{W} 12: 81.1 \%$ versus $80.0 \%$, respectively $(\mathrm{E}(\mathrm{SE})=1.08(6.60) \%, 95 \% \mathrm{CI}=[-11.86 ; 14.02])$.

\section{- Office standing SBP/DBP at 3 min at each last post-baseline value over the W0-W12 period}

A decrease in the office standing SBP/DBP at 3 min was observed in both treatment groups from baseline to each last post-baseline value at W4, W8, and W12. The changes were similar in both treatment groups. The full effect on the office supine SBP was reached at W4 and maintained until W12, in both treatment groups, in the FAS:

- W4: $\mathrm{SBP}=-19.8 \pm 13.0 \mathrm{mmHg}$ in the Per/Ind/Aml group versus $-15.5 \pm 12.8 \mathrm{mmHg}$ in the Per/Ind + Aml group $(\mathrm{E}(\mathrm{SE})=-3.47(1.89) \mathrm{mmHg}, 95 \% \mathrm{CI}=[-7.21 ; 0.27]) ; \mathrm{DBP}=-13.6 \pm 11.0 \mathrm{mmHg}$ versus $-11.4 \pm 10.9$, respectively $(\mathrm{E}(\mathrm{SE})=-1.42(1.52) \mathrm{mmHg}, 95 \% \mathrm{CI}=[-4.42 ; 1.58])$.

- W8: $\mathrm{SBP}=-18.9 \pm 13.2 \mathrm{mmHg}$ versus $-21.6 \pm 15.5 \mathrm{mmHg}$, respectively $(\mathrm{E}(\mathrm{SE})=3.56(2.12) \mathrm{mmHg}$, $95 \% \mathrm{CI}=[-0.64 ; 7.76]) ; \mathrm{DBP}=-14.4 \pm 11.3 \mathrm{mmHg}$ versus $-15.6 \pm 11.8 \mathrm{mmHg}$, respectively $(\mathrm{E}(\mathrm{SE})=2.27(1.60) \mathrm{mmHg}, 95 \% \mathrm{CI}=[-0.89 ; 5.43])$.

- W12: $\mathrm{SBP}=-20.3 \pm 14.7 \mathrm{mmHg}$ versus $-18.5 \pm 13.9 \mathrm{mmHg}$, respectively $(\mathrm{E}(\mathrm{SE})=-0.78(2.00)$ $\mathrm{mmHg}, 95 \% \mathrm{CI}=[-4.73 ; 3.16]) ; \mathrm{DBP}=-15.3 \pm 10.4 \mathrm{mmHg}$ versus $-12.6 \pm 9.9 \mathrm{mmHg}$, respectively $(\mathrm{E}(\mathrm{SE})=-1.95(1.43) \mathrm{mmHg}, 95 \% \mathrm{CI}=[-4.79 ; 0.89])$.

In the PPS, results of the secondary criteria were in the same line. 


\section{SUMMARY - CONCLUSIONS (Cont'd) SAFETY RESULTS}

\section{- Emergent adverse events}

Emergent adverse events in the Safety Set $(\mathrm{N}=148)$

\begin{tabular}{llcc}
\hline Patients having reported at least one & & $\begin{array}{c}\text { Per/Ind/Aml } \\
(\mathbf{N}=\mathbf{7 5})\end{array}$ & $\begin{array}{c}\text { Per/Ind + Aml } \\
\mathbf{( N = 7 3 )}\end{array}$ \\
\hline $\begin{array}{l}\text { Emergent adverse event (EAE) } \\
\text { Treatment-related EAE }\end{array}$ & $\mathrm{n}(\%)$ & $14(18.7)$ & $16(21.9)$ \\
EAE: hypotension & $\mathrm{n}(\%)$ & $3(4.0)$ & $5(6.8)$ \\
$\begin{array}{l}\text { Emergent calculated hypotension } \\
\text { orthostatic* }\end{array}$ & $\mathrm{n}(\%)$ & - & $1(1.4)$ \\
$\begin{array}{l}\text { Serious EAE } \\
\text { Treatment-related serious EAE }\end{array}$ & - & $1(1.4)$ \\
$\begin{array}{l}\text { EAE leading to treatment withdrawal } \\
\text { Serious EAE }\end{array}$ & $\mathrm{n}(\%)$ & $1(1.3)$ & - \\
$\quad \begin{array}{l}\text { Treatment-related EAE } \\
\text { Treatment-related serious EAE }\end{array}$ & $\mathrm{n}(\%)$ & $1(1.3)$ & $1(1.4)$ \\
Patients who died & $\mathrm{n}(\%)$ & - & - \\
& $\mathrm{n}(\%)$ & - & - \\
\hline
\end{tabular}

$N$ : total number of patients in each considered treatment group; $n$ : number of patients affected; \%: $\left(n^{*} 100 / \mathrm{N}\right)$; *: reduction of at least $20 \mathrm{mmHg}$ for SBP and/or $10 \mathrm{mmHg}$ for DBP at 1 and 3 minutes after standing-up compared to supine position, detected at post-baseline visit but not present at baseline; EAE: emergent adverse event.

Emergent adverse events (EAEs) were reported in 14/75 patients (18.7\%) in the Per/Ind/Aml group and $16 / 73$ patients $(21.9 \%)$ in the Per/Ind + Aml group, respectively.

The SOCs most frequently affected in the Per/Ind/Aml group was Infections and infestations (7/75 patients, (9.3\%) in the Per/Ind/Aml group and 2/73 patients (2.7\%) in the Per/Ind + Aml group), and in the Per/Ind + Aml group: Investigations (1/75 patients (1.3\%) and 6/73 patients (8.2\%), respectively).

EAEs more frequently reported were in the Per/Ind/Aml group:

- Nasopharyngitis: $2 / 75$ patients (2.7\%) in the Per/Ind/Aml group versus $1 / 73$ patient (1.4\%) in the Per/Ind + Aml group,

- Hypokalaemia: 2/75 patients (2.7\%) only in the Per/Ind/Aml group.

EAEs more frequently reported were in the Per/Ind + Aml group: blood potassium increased, blood urea increased, and hyperuricaemia (2/73 patients only in the Per/Ind + Aml group (2.7\% each preferred term)).

It should be noted that the following EAEs listed in the Summary of Product Characteristics (SmPCs): hypokalaemia, hyperkalaemia, peripheral oedema, or cough were either not reported at all (hyperkalaemia, peripheral oedema, cough) or uncommon (hypokalaemia: 2 patients in the Per/Ind/Aml group).

A particular focus was done on emergent hypotension and orthostatic hypotension. One patient reported an emergent hypotension in the Per/Ind + Aml group, but none of the patients was affected by a symptomatic orthostatic hypotension. This EAE was not serious, not considered as treatment-related according to the investigator, and recovered. Emergent calculated orthostatic hypotension, defined according to a mathematic rule (reduction of at least $20 \mathrm{mmHg}$ for SBP and/or $10 \mathrm{mmHg}$ for DBP at 1 and 3 minutes after standing-up compared to supine position, detected at post-baseline visit but not present at baseline) affected one patient only in the Per/Ind + Aml group (asymptomatic). Most of the EAEs were rated mild, with a lower rate in the Per/Ind/Aml group than in the Per/Ind + Aml group $557.9 \%$ of the total of the EAEs versus $75.0 \%$, respectively). A total of 3 EAEs severe were reported in one patient in the Per/Ind/Aml group: atrial fibrillation, pneumonia, dyspnoea. These EAEs were serious, not considered as treatment-related according to the investigator's opinion, did not lead to patient's withdrawal, and resolved.

EAEs considered as treatment-related according to the investigator's opinion, were in accordance with those listed in the SmPC of perindopril, indapamide, and amlodipine. Few treatment-related EAEs were reported in $3 / 75$ patients $(4.0 \%)$ in the Per/Ind/Aml group versus 5/73 patients (6.8\%) in the Per/Ind + Aml group. No preferred term was affected more than once, except hypokalaemia in $2 / 75$ patients $(2.7 \%)$ in the $\mathrm{Per} / \mathrm{Ind} / \mathrm{Aml}$ group. None of these treatment-related adverse events was serious. 


\section{SUMMARY - CONCLUSIONS (Cont'd) SAFETY RESULTS (Cont'd)}

EAEs leading to a premature patient's treatment withdrawal were reported in one patient in each treatment group: hypertension in the Per/Ind/Aml group and rash in the Per/Ind + Aml group. These EAEs were nonserious, and recovered. The rash was considered by the investigator as treatment-related.

Most of the EAEs recovered: $84.2 \%$ of the total EAEs in the Per/Ind/Aml group and $75.0 \%$ in the Per/Ind + Aml group.

A total of 3 serious EAEs were reported in one patient in the Per/Ind/Aml group: atrial fibrillation, pneumonia, dyspnoea. These EAEs were severe (see above for details).

None of the patients died during the study.

- Laboratory tests

For biochemistry, the rate of patients affected by PCSA values was low in both treatments groups $(<5 \%)$. PCSAs values were mostly detected for creatinine clearance (low value, whatever the used formula) in $3 / 71$ patients $(4.2 \%)$ in the Per/Ind/Aml group and 3/68 patients $(4.4 \%)$ in the Per/Ind + Aml group. All these patients were those that had at baseline a creatinine clearance $<90 \mathrm{~mL} / \mathrm{min}$ or $\mathrm{mL} / \mathrm{min} / 1.73 \mathrm{~m}^{2}$, and were aged $\geq 60$ years except one patient younger than 60 years in the Per/Ind + Aml group.

A decrease in the glomerular filtration rate $>25 \%$ from baseline (considered as relevant by the KDIGO Guidelines (2012)) was observed in $2 / 3$ patients in the Per/Ind/Aml group (low PCSA value), of which one patient had a decrease $>30 \%$. This effect well described in the TRIPLIXAM ${ }^{\circledR}$ SmPC was probably due to a transitory functional renal insufficiency at the beginning of the administration of the treatments.

In the Per/Ind/Aml group, among the 3 patients with PCSA values for creatinine clearance, 2 had low values that were not any more PCSA at $\mathrm{W} 12$, and returned on treatment to a level comparable to that observed at baseline (including the patient with a relative decrease from baseline $>30 \%$ ). These 3 patients did not present any PCSA for other biochemistry parameter.

In the Per/Ind + Aml group: 3 patients had a low PCSA value for creatinine clearance, with a decrease from baseline $<25 \%$. For $1 / 3$ patients, the value returned to a level $>60 \mathrm{~mL} / \mathrm{min}$ or $\mathrm{mL} / \mathrm{min} / 1.73 \mathrm{~m}^{2}$ at W12. One of the 3 patients had also a high PCSA value for urea (of which one was reported as adverse event), but the other patients did not present any PCSA for other parameters of the renal function (potassium, urea, creatinine). One additional patient (4.4\%) had high PCSA value for uric acid (reported as adverse event).

For haematology, 2 low PCSAs values were detected for haematocrit in 2 patients, 3.0\% in the Per/Ind + Aml group.

- Vitals signs and ECG

Neither clinically relevant changes nor between-group difference over time were detected. None of the ECG abnormalities detected at post-baseline visits was considered as clinically significant by the investigator.

\section{CONCLUSION}

This international open Phase III study over 12 weeks, aimed to assess the efficacy and safety of the single-pill combination of the fixed dose combination of perindopril $5 \mathrm{mg} / \mathrm{indapamide}$ $1.25 \mathrm{mg} /$ amlodipine $5 \mathrm{mg}$ versus free dual therapy (perindopril/indapamide and amplodipine taken separately), at the same dose, in patients with essential hypertension, uncontrolled by their usual antihypertensive treatment (monotherapy at maximal dose or a single dose of dual therapy). A decrease in SBP and DBP was reached at 1 month of treatment and maintained until 3 months, with the single-pill combination and the free dual therapy. Most of the patients in both treatment groups responded to the antihypertensive therapy, and reached a BP control, observed at 1 month of treatment and until 3 months. No relevant between-group difference was observed.

The single-pill combination and the free dual therapy were well tolerated and the safety profile was in accordance with the SmPCs of perindopril, indapamide, and amlodipine. No relevant between-group difference was observed.

Date of the report: 26 February 2015

Version of the report: Final version 